\title{
Reconstruction of chronic Achilles tendon rupture using the semitendinosus tendon : a case report
}

\author{
Makoto Takeuchi, Naoto Suzue, Tetsuya Matsuura, Kosaku Higashino, \\ Toshinori Sakai, Daisuke Hamada, Tomohiro Goto, Yoichiro Takata, \\ Toshihiko Nishisho, Yuichiro Goda, Ryosuke Sato, Ichiro Tonogai, \\ Kazuaki Mineta, and Koichi Sairyo \\ Department of Orthopedics, the University of Tokushima, Tokushima, Japan
}

\begin{abstract}
Achilles tendon rupture is a common trauma requiring surgical management. For chronic Achilles tendon rupture in particular, reconstructive surgery is desirable and several methods have been described. Here we present a case of chronic Achilles tendon rupture reconstructed using the semitendinosus tendon because of the difficulty in pulling down the proximal stump to reach the distal stump and due to an insufficient margin for hooking a suture to the distal stump. Postoperatively, the patient had a fully functional tendon and resumed his normal activities of daily living. Using this surgical technique, we expect favorable outcomes in cases of Achilles tendon rupture. J. Med. Invest. 61 : 417-420, August, 2014
\end{abstract}

Keywords : Achilles tendon reconstruction, chronic rupture, semitendinosus tendon

\section{INTRODUCTION}

Achilles tendon rupture represents more than $40 \%$ of all tendon ruptures requiring surgical management (1). Although it can be managed conservatively or operatively, the re-rupture rate of operative treatment is typically lower than that of conservative therapy $(2,3)$. In cases such as chronic Achilles tendon rupture in which the tendon has greatly degenerated or a large gap is present at the rupture site, operative repair can be more difficult than for acute rupture ; therefore, reconstructive surgery of the Achilles tendon is more desirable (4). While several techniques for Achilles tendon reconstruction have been described, the superiority of one

Received for publication April 4, 2014 ; accepted May 15, 2014.

Address correspondence and reprint requests to Tetsuya Matsuura, MD and PhD, Assistant Professor, Department of Orthopedics, the University of Tokushima, 3-18-15 Kuramoto, Tokushima 770-8503, Japan and Fax : +81-88-633-0178. technique over another has not been demonstrated and optimal surgical management of Achilles tendon rupture remains controversial (5). Among the available options, the semitendinosus tendon has been used for open reconstruction of chronic Achilles tendon rupture with encouraging results (6). In this report, we describe a case of chronic Achilles tendon reconstruction using the semitendinosus tendon with a favorable outcome.

\section{CASE REPORT}

A 48-year-old man noticed left heel pain while carrying a $30-\mathrm{kg}$ load and consulted a local doctor who provided non-surgical treatment with steroid injections. His symptoms improved temporarily ; however, 1 month later, he injured his left heel through forced inversion stress when putting on his shoes and was unable to stand because of severe left heel pain. He received first aid at an emergency 
hospital, where Achilles tendon injury was noted. He was treated conservatively with plaster for 2 months and thereafter received an orthosis. He then strained his left foot, exacerbating his left heel pain. Three months after the initial injury, he was referred to our hospital for further examination.

The first physical examination revealed no tenderness but gap was palpable in the insertion of the Achilles tendon on the calcaneus, and the Thompson test revealed significantly weaker planter flexion of the left ankle compared with the right one. Plain radiography and computed tomography of the left ankle showed calcification in the insertion of the Achilles tendon into the calcaneus (Figure 1), and T2-weighted and short time inversion recovery images revealed a high-intensity area in the same region (Figure 2).

We diagnosed the present case as partial chronic rupture of the left Achilles tendon. Written informed

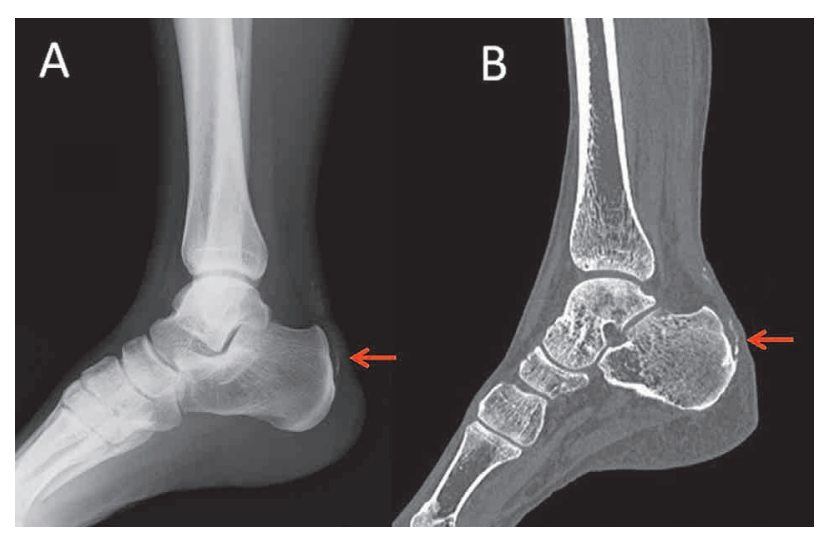

Figure 1 Sagittal view of the left ankle showing calcification in the insertion of the Achilles tendon into the calcaneus (red arrows). (A) Plain radiography. (B) Computed tomography.

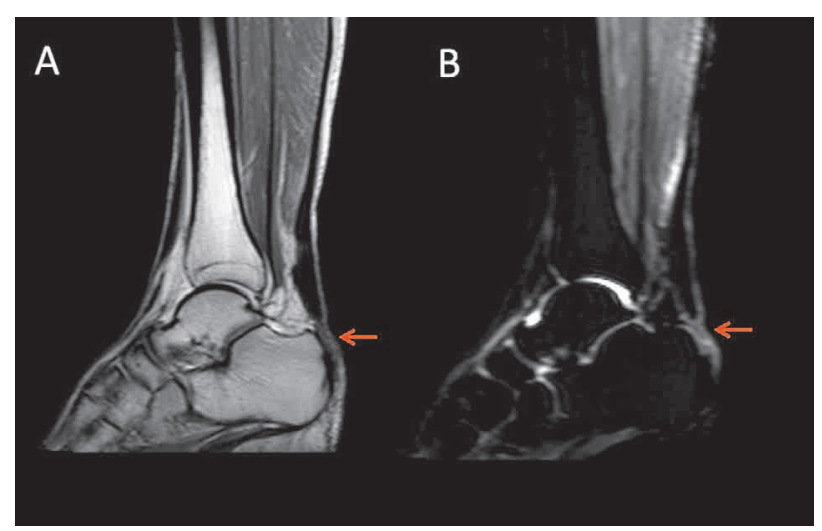

Figure 2 Sagittal view magnetic resonance imaging of the left ankle showing a high-intensity lesion in the insertion of the Achilles tendon into the calcaneus (red arrows). (A) T2-weighted imaging. (B) Short time inversion recovery imaging. consent was obtained from the patient and performed open surgery under general anesthesia with the patient in the prone position. The Achilles tendon approximately $1 \mathrm{~cm}$ proximal to the insertion on the calcaneus was ruptured, and the gap between the ends of the ruptured tendon was approximately $1.5 \mathrm{~cm}$, while the medial part of the tendon showed mostly sustained continuity (Figure 3 ). We opted to use the semitendinosus tendon for reconstruction because it was difficult to pull down the proximal stump to reach the distal stump and there was an insufficient margin for hooking a suture to the distal stump. We harvested the semitendinosus tendon from the left leg using a tendon stripper. To prevent of neurological injury, keep the knee flexed during tendon harvest and ensuring that all adhesions are cleared. Then we made a bone tunnel parallel to the articular surface of the ankle joint in the calcaneus with a $4.5-\mathrm{mm}$ diameter drill. The semitendinosus tendon was passed through the bone tunnel and crossed in the regions proximal to the distal stump and proximal stump of the Achilles tendon (Figure 4). The semitendinosus tendon was sutured to the proximal stump of the Achilles tendon

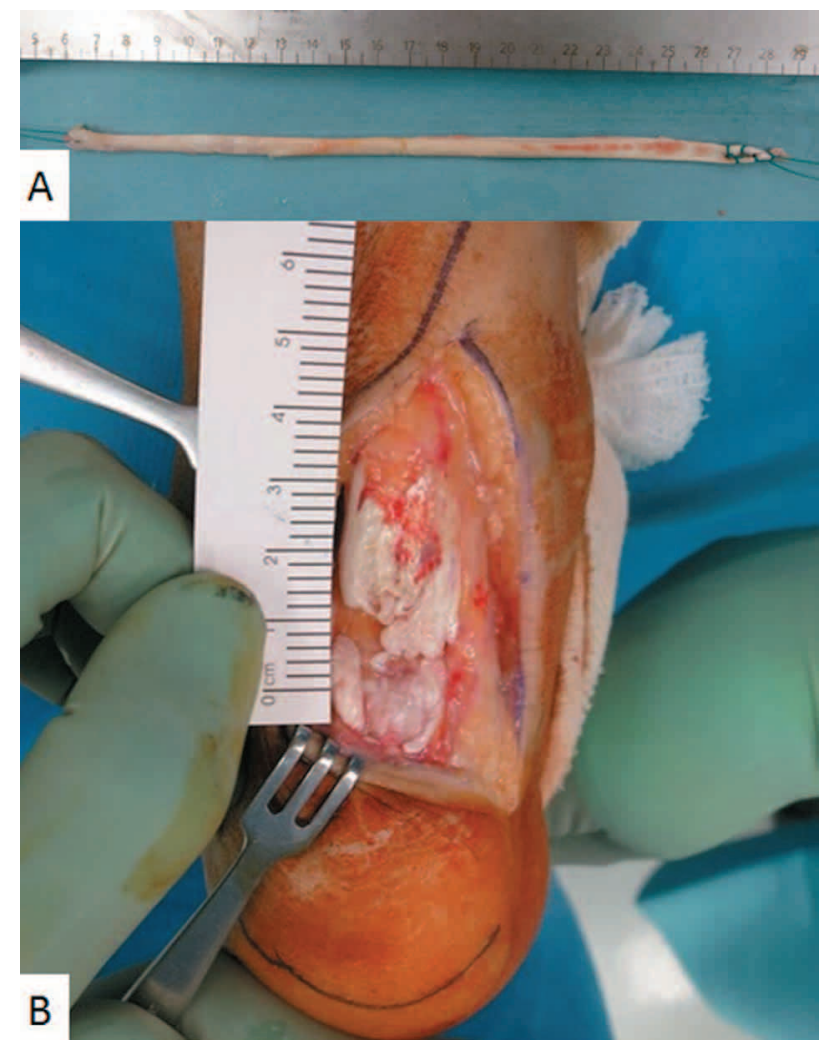

Figure 3 Intraoperative photographs. (A) Harvested semitendinosus tendon sutured with 1-0 ethibond using the baseball suturing technique at both ends. (B) Left Achilles tendon rupture with the medial part of the tendon showing mostly sustained continuity with a gap of approximately $1.5 \mathrm{~cm}$ between the ends. 


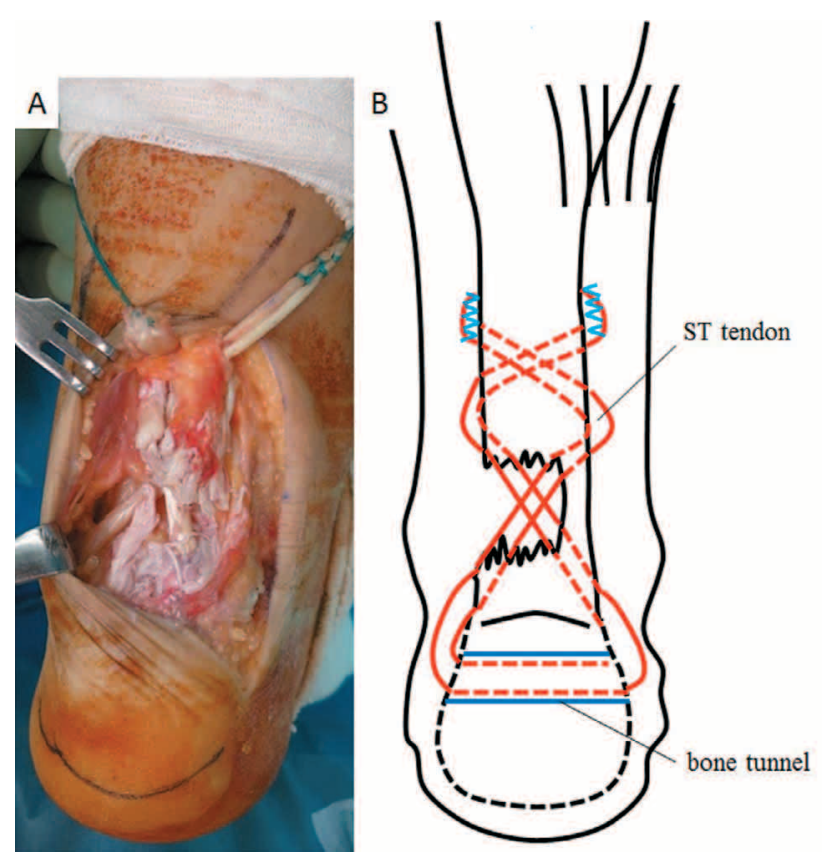

Figure 4 Surgical outcome. (A) Intraoperative photograph showing the reconstructed left Achilles tendon. (B) Diagrammatic representation of the reconstruction.

with 1-0 ethibond sufficient for the tension placed on the semitendinosus tendon by plantar flexion and dorsiflexion of the ankle.

Postoperatively, the operated ankle remained in a cast for the first 4 weeks. After the cast was removed, progressive load training on the operated ankle was performed while using an ankle foot orthosis (The Ultra CTS ${ }^{\mathrm{TM}}$ Ankle Brace, Breg, Carlsbad, CA), which the patient wore for 3 months after the surgery. Nine weeks after the surgery, the patient was allowed full weight bearing. At the 8-month follow up, he had a fully functional tendon and had resumed his normal activities of daily living. Sensory change over the front of the knee was not present in this case.

\section{DISCUSSION}

Several techniques for Achilles tendon reconstruction have been described, including turndown flap, tendon transfer, tendon graft, and augmentation with synthetic materials (6). However, which of these provides the best surgical management of chronic Achilles tendon rupture has not been established (5). Tendon transfer techniques have been widely used with good results but can be associated with loss of function. Indeed, transfer of the flexor hallucis longus tendon results in a permanent decrease in the strength of the great toe flexor (7), whereas transfer of the flexor digitorum longus tendon affects push-off strength $(8,9)$. Furthermore, a V-Y plasty may be undertaken $(10,11)$, but it alters permanently the ratio between the tendon and muscle length.

To overcome these problems, we chose to use hamstring tendons to reconstruct chronic rupture of the Achilles tendon in the present case. Hamstring tendons are currently used as a free graft for anterior cruciate ligament reconstruction because of the low risk of donor site morbidity, fast recovery, and easy harvest $(12,13)$. Moreover, they are relatively long tendons, enabling reconstruction of Achilles tendon continuity, even in cases of chronic rupture with a wide gap between the stumps (14).

In some cases of Achilles tendon rupture, a large gap $(>6 \mathrm{~cm})$ exists between the ends of the tendon, despite maximal plantar flexion of the ankle and traction on the Achilles tendon stumps, and the local tendons are insufficient to bridge the gap. Maffulli et al. suggested that in such instances reconstruction using the ipsilateral hamstring tendon is a suitable option (15-18). In the present case, although the gap between the ends of the ruptured Achilles tendon was approximately $1.5 \mathrm{~cm}$, transfer of the semitendinosus tendon was selected because of the difficulty in pulling down the proximal stump to reach the distal stump and due to an insufficient margin for hooking a suture to the latter stump, and a good outcome was achieved. Also, it is desirable to reinforce tendons in patients whose stump have calcification because of the accelerated collagen degeneration.

There is a perception that Achilles tendon reconstruction with the hamstrings method is associated with lower postoperative morbidity. However, this technique is not without complications. This includes the sensory deficits of the saphenous nerve and its infrapatellar branch. It is important to keep the knee flexed during tendon harvest to prevent neurological injury (19). In this case, we performed to do so, sensory change was not present.

Reconstructing a chronic ruptured Achilles tendon using the semitendinosus tendon can offer satisfactory results even in the presence of a relatively small gap $(<6 \mathrm{~cm})$ when matching up the distal stamp and proximal one stumps of a ruptured Achilles tendon is very difficult. We anticipate favorable outcomes with this surgical technique. 


\section{CONFLICT OF INTEREST}

None of the authors received any funding support to complete this report. Also, none of the authors have any conflicts of interest to declare.

\section{REFERENCES}

1. Jozsa L, Kvist M, Balint BJ, Reffy A, Järvinen $\mathrm{M}$, Lehto M, Barzo M : The role of recreational sport activity in Achilles tendon rupture : a clinical, pathoanatomical, and sociological study of 292 cases. Am J Sports Med 17 : 338-343, 1989

2. Wallace RG, Traynor IE, Kernohan WG, Eames $\mathrm{MH}$ : Combined conservative and orthotic management of acute ruptures of the Achilles tendon. J Bone Joint Surg Am 86 : 108-202, 2004

3. Lawrence SJ, Grau GF : Management of acute Achilles tendon ruptures. Orthopedics $27: 579$ 581, 2004

4. Uchida R, Natsuume T, Yoneda K, Fuji T : Repair of achilles tendon rupture using autologous semitendinosus graft in a kidney transplant recipient. J Foot Ankle Surg 53 : 194-198, 2014

5. Wong J, Barrass V, Maffulli N : Quantitative review of operative and nonoperative management of Achilles tendon ruptures. Am J Sports Med 30 : 565-575, 2002

6. Sarzaeem MM, Lemraski MM, Safdari F : Chronic Achilles tendon rupture reconstruction using a free semitendinosus tendon graft transfer. Knee Surg Sports Traumatol Arthrosc 20 : 1386-1391, 2012

7. Longo UG, Lamberti A, Maffulli N, Denaro V : Tendon augmentation grafts : a systematic review. Br Med Bull 94 : 165-188, 2010

8. Wapner KL, Pavlock GS, Hecht PJ, Naselli F, Walther R : Repair of chronic Achilles tendon rupture with flexor hallucis longus tendon transfer. Foot Ankle 14 : 443-449, 1993

9. Ferris L, Sharkey NA, Smith TS, Matthews DK : Influence of extrinsic plantar flexors on forefoot loading during heel rise. Foot Ankle Int $16: 464-473,1995$

10. Abraham E, Pankovich AM : Neglected rupture of the Achilles tendon : treatment by V-Y tendinous flap. J Bone Joint Surg Am 57 : 253-255, 1975

11. Leitner A, Voigt C, Rahmanzadeh R : Treatment of extensive aseptic defects in old Achilles tendon ruptures : methods and case reports. Foot Ankle 13 : 176-180, 1992

12. Jacob HA : Forces acting in the forefoot during normal gait: an estimate. Clin Biomech 16 : 783-792, 2001

13. Leys T, Salmon L, Waller A, Linklater J, Pinczewski L : Clinical results and risk factors for reinjury 15 years after anterior cruciate ligament reconstruction : a prospective study of hamstring and patellar tendon grafts. Am J Sports Med 40 : 595-605, 2012

14. Papandrea P, Vulpiani MC, Ferretti A, Conteduca F : Regeneration of the semitendinosus tendon harvested for anterior cruciate ligament reconstruction : evaluation using ultrasonography. Am J Sports Med 28 : 556-561, 2000

15. Maffulli N, Ajis A : Management of chronic ruptures of the Achilles tendon. J Bone Joint Surg Am 90 : 1348-1360, 2008

16. Maffulli N, Longo UG, Spiezia F, Denaro V : Free hamstrings tendon transfer and interference screw fixation for less invasive reconstruction of chronic avulsions of the Achilles. Knee Surg Sports Traumatol Arthrosc 18 : 269-273, 2010

17. Maffulli N, Longo UG, Gougoulias N, Denaro $\mathrm{V}$ : Ipsilateral free semitendinosus tendon graft transfer for reconstruction of chronic tears of the Achilles tendon. BMC Musculoskelet Disord $9: 100,2008$

18. Maffulli N, Spiezia F, Longo UG, Denaro V : Less-invasive reconstruction of chronic Achilles tendon ruptures using a peroneus brevis tendon transfer. Am J Sports Med 38 : 2304-2312, 2010

19. Spicer DD, Blagg SE, Unwin AJ, Allum RL: Anterior knee symptoms after four-strand hamstring tendon anterior cruciate ligament reconstruction. Knee Surg Sports Traumatol Arthrosc $8: 286$ - 289,2000 